\title{
Wave-packet dynamics in quantum wells
}

Kuznetsov, A. V.; Sanders, G. D.; Stanton, C. J.

Published in:

Physical Review B Condensed Matter

Link to article, DOI:

10.1103/PhysRevB.52.12045

Publication date:

1995

Document Version

Publisher's PDF, also known as Version of record

Link back to DTU Orbit

Citation (APA):

Kuznetsov, A. V., Sanders, G. D., \& Stanton, C. J. (1995). Wave-packet dynamics in quantum wells. Physical Review B Condensed Matter, 52(16), 12045-12055. https://doi.org/10.1103/PhysRevB.52.12045

\section{General rights}

Copyright and moral rights for the publications made accessible in the public portal are retained by the authors and/or other copyright owners and it is a condition of accessing publications that users recognise and abide by the legal requirements associated with these rights.

- Users may download and print one copy of any publication from the public portal for the purpose of private study or research.

- You may not further distribute the material or use it for any profit-making activity or commercial gain

- You may freely distribute the URL identifying the publication in the public portal

If you believe that this document breaches copyright please contact us providing details, and we will remove access to the work immediately and investigate your claim. 


\title{
Wave-packet dynamics in quantum wells
}

\author{
A. V. Kuznetsov* \\ Department of Physics, University of Florida, Gainesville, Florida 32611 \\ G. D. Sanders ${ }^{\dagger}$ and C. J. Stanton ${ }^{\dagger}$ \\ Mikroelektronik Centret, Danmarks Tekniske Universitet, DK-2800 Lyngby, Denmark \\ (Received 21 April 1995)
}

\begin{abstract}
It has been recently recognized that in bulk semiconductors the displacement current caused by ultrafast optical generation of "polarized pairs" in the applied dc field is an important mechanism of charge transport in addition to the usual transport current. In quantum-well systems, this polarized pair creation is thought to be the only source of photocurrent at the early stages of photoexcitation since the bulklike transport current is inhibited by the barriers. In this work we perform a full quantummechanical analysis of ultrafast optical excitation in a dc-biased quantum well. We take into account the multiple transitions that become allowed in the dc field which breaks the $\Delta n=0$ selection rule. As a result, the carriers are created as wave packets formed by coherent superposition of several eigenstates. When the characteristic size of these wave packets (coherence length) is much larger than the well width (for long pulses and/or narrow wells), we recover the polarized pairs behavior of the photocurrent. For shorter pulses, when the coherence length becomes comparable to the well width, the photocurrent exhibits quantum beats. Finally, for very short pulses (around $10 \mathrm{fs}$ ) we find that the carriers in a quantum well can behave as an ensemble of classical particles and produce a transportlike photocurrent.
\end{abstract}

\section{INTRODUCTION}

Ultrafast response in semiconductor quantum-well systems has been the subject of much experimental and theoretical attention over the past few years. ${ }^{1}$ At present, a variety of complementary experimental techniques for studying quantum-confined systems on a femtosecond time scale is available. Typically, a multiple quantum well or a superlattice sample is optically excited with a femtosecond laser, and the resulting evolution of photoexcited carriers is time resolved by measuring optical [pump and probe, four-wave mixing (FWM)] (Refs. 1 and 2) or transport ( $\mathrm{THz}$ radiation) (Refs. $2-7$ ) properties of the sample at different time delays.

A distinct class of ultrafast processes in quantum-well systems arises when a dc electric field is applied to the sample in the growth direction. In quantum-confined systems, the field-induced separation of photoexcited electrons and holes is hampered by the barriers. In a superlattice, the carriers can tunnel through the barriers and move according to the dispersion of the minibands, which can lead to Bloch oscillations ${ }^{8}$ of the photocurrent. Such oscillations have been observed in superlattice structures. $^{5-7}$ When the well spacing is large, tunneling is suppressed, and one has a multiple-quantum-well system as opposed to a superlattice. In quantum wells, the transport current in the growth direction is completely inhibited by the barriers.

However, as it was experimentally ${ }^{3,4}$ and theoretical$1 y^{9,10}$ demonstrated, a different type of photocurrent is still present in photoexcited single quantum wells. Because the quantum-confined states are polarized by the applied dc field, electron-hole pairs are created by the optical pulse with a nonzero dipole moment. ${ }^{9}$ The quantum well acquires a time-dependent dipole moment (dielectric polarization) which is proportional to the total number of pairs in the well [Fig. 1(a)]. The time derivative of the polarization is the displacement current which has been observed by measuring the transient electromagnetic radiation it produces. $3,4,11,12$ For excitation below the band gap, the levels are populated by virtual carriers which disappear after the pulse ${ }^{13,14}$ [Fig. 1(b)], but the proportionality of the dipole moment to the number of pair still holds in this case. In both cases, the photocurrent is only present during the optical pulse. This feature has been described as virtual photoconductivity, ${ }^{14}$ and opens a

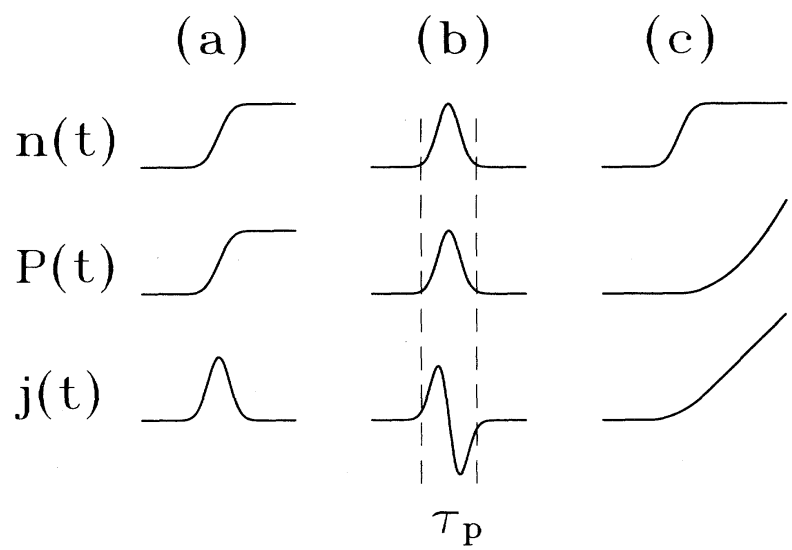

FIG. 1. Schematic representation of time-dependent carrier density, dielectric polarization, and photocurrent after ultrafast optical excitation for (a) quantum-confined sytem, excitation above the band gap (real carriers); (b) virtual carriers (excitation below the band gap); and (c) real carriers in a bulk sample. 
possibility for the generation of ultrashort electric current pulses. ${ }^{15}$

A necessary condition for the instantaneous, polarized-pair displacement photocurrent to occur is that the well be narrow enough, since in a very wide bulklike system the dynamics of photocurrent should be quite different. In a three-dimensional (3D) system, the photocurrent is usually dominated by a transport component that results from field-induced separation of real, photoexcited electrons and holes, and does not vanish after the pulse is over ${ }^{10-12}$ [Fig. 1(c)]. As the width of the well increases, one can expect a smooth transition from an adiabatic 2D response depicted in Figs. 1(a) and 1(b) to the slower bulklike current dynamics of Fig. 1(c). From both theoretical and practical standpoints, it is desirable to develop a quantitative method that can predict which of the possible types of photocurrent response will be present for a given system and excitation conditions. The development of such an approach is the goal of this paper.

Usually, carriers in a microstructure are considered to be quantum confined if the energy spacing between quantized levels is greater than collisional broadening of the levels. ${ }^{1,6}$ (We will not consider excitons here for which the criterion is different. ${ }^{13}$ ) In the present paper, we investigate the $2 \mathrm{D}-3 \mathrm{D}$ transition in the ultrafast photocurrent, and identify the relevant physical parameters affecting it. Our results indicate that the above criterion does not work for excitation pulses shorter than the relevant relaxation times. We find that the critical width of the well beyond which it behaves essentially as a 3D system depends on the optical pulse duration and the photon energy, and is different for electrons and the holes. We formulate simple quantitative criteria for different types of response in terms of the transient localization concept introduced in Ref. 10. We demonstrate that for a well to behave like a $2 \mathrm{D}$ system, the coherence length for photoexcited carriers has to be greater than the width of the well.

The paper is organized as follows: Section II describes the theoretical model that we use to describe the quantum well and its optical and transport properties. We present our results for the photocurrent and related quantities in Sec. III, where we also give a simple physical interpretation of our results, and discuss their relevance for various experimental situations. The final Sec. IV contains concluding remarks.

\section{GENERAL THEORY}

\section{A. Quantum-well structure and electronic states}

For simplicity, we consider a quantum well with infinite barriers at $z= \pm L / 2$ (perpendicular to the [001] direction). The wave functions for confined carriers in a quantum well $\varphi_{n, \mathbf{k}}^{\alpha}(\mathbf{r})$ are obtained by multiplying the periodic part of the band-edge bulk Bloch states, $u^{\alpha}(\mathbf{r})$, by a slowly varying envelope function. Thus

$$
\varphi_{n, \mathbf{k}}^{\alpha}(\mathbf{r})=\frac{e^{i \mathbf{k} \cdot \mathbf{r}}}{\sqrt{A}} F_{n}^{\alpha}(z) u^{\alpha}(\mathbf{r})
$$

where $\alpha=c, h$, and $l$ labels the carrier type (electron, heavy hole, or light hole, respectively), and $n$ labels the subband index. The complete envelope function is the product of a plane wave (with cross-sectional area $A$ and wave vector $k$ ) describing translational motion in the $x-y$ plane multiplied by a slowly varying function $F_{n}^{\alpha}(z)$, which describes the confined motion of the carriers along the $z$ axis.

Inside the quantum well, the envelope functions $F_{n}^{\alpha}(z)$ satisfy the one-dimensional effective-mass Schrödinger equation

$$
\left[-\frac{\hbar^{2}}{2 m_{\alpha \perp}} \frac{d^{2}}{d z^{2}}+V_{\alpha}(z)\right] F_{n}^{\alpha}(z)=E_{n}^{\alpha} F_{n}^{\alpha}(z),
$$

where $m_{\alpha \perp}$ is the effective mass along the $z$ direction.

The quantum-well band structure consists of a set of parabolic subbands. For electrons the subband energies are

$$
E_{n}^{c}(k)=E_{g}+E_{n}^{c}+\frac{\hbar^{2} k^{2}}{2 m_{c \|}},
$$

and for heavy and light holes the subband energies are

$$
E_{n}^{h}(k)=-E_{n}^{h}-\frac{\hbar^{2} k^{2}}{2 m_{h \|}}, \quad E_{n}^{l}(k)=-E_{n}^{l}-\frac{\hbar^{2} k^{2}}{2 m_{l \|}} .
$$

The effective masses in the $x-y$ plane are denoted $m_{\alpha \|}$. For GaAs, the electron mass is isotropic with $m_{c \perp}=m_{c \|}=0.067 m_{0}$. The hole masses can be expressed through the Luttinger parameters ${ }^{16,17}$ which for GaAs results in $m_{h \perp}=0.408 m_{0}$ and $m_{h \|}=0.109 m_{0}$ effective masses for the heavy holes, while for the light-holes we have $m_{l \perp}=0.087 m_{0}$ and $m_{l \|}=0.244 m_{0}$. In this model, for simplicity, we have neglected the off-diagonal terms in the Luttner Hamiltonian for the valence bands. The hole masses are therefore isotropic in the plane of the wells, but differ in the $z$ direction.

The potential $V_{\alpha}(z)$ which appears in the effectivemass Schrödinger equation is the potential due to the dc electric field $F$. For electrons $V_{c}(z)=e F z$, and for holes $V_{h}(z)=V_{l}(z)=-e F z$.

We solve the Schrödinger equation using finite differences on an evenly spaced mesh. We let $f_{i}=F_{n}^{\alpha}\left(z_{i}\right)$ denote the wave function, and $v_{i}=V_{\alpha}\left(z_{i}\right)$ denote the potential where $i=0, \ldots, N+1$ labels the mesh points. In a quantum well with infinite barriers, the wave function vanishes at the boundary so that $f_{0}=f_{N+1}=0$. Approximating the second derivative of the envelope function at each interior mesh point as

$$
\frac{d^{2} f_{i}}{d z^{2}}=\frac{f_{i+1}-2 f_{i}+f_{i-1}}{h^{2}}, \quad i=1, \ldots, N,
$$

where $h$ is the spacing between mesh points, we obtain a trigonal matrix eigenvalue problem of $\operatorname{order} N$, whose eigenvalues are the energies $E_{n}^{\alpha}$ and whose eigenvectors are the envelope functions $F_{n}^{\alpha}\left(z_{i}\right)$ at the interior mesh points.

In Fig. 2 we show the lowest-lying energy levels and envelope functions of a 200 - $\AA$-wide quantum well in a dc field of $50 \mathrm{kV} / \mathrm{cm}$. The electric field points from left to 

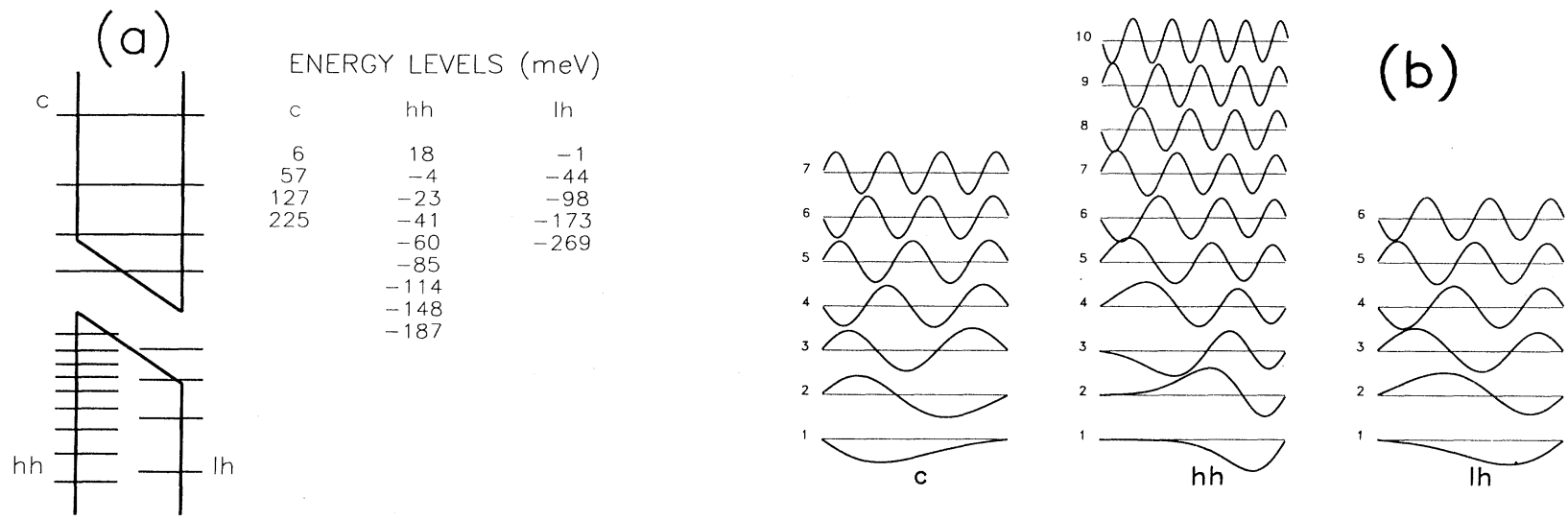

FIG. 2. Energy levels (a) and wave functions (b) for a 20-nm quantum well with infinite barriers in a $50-\mathrm{kV} / \mathrm{cm}$ electric field calculted by the procedure discussed in the text.

right in the figure so the lowest-lying electron state is shifted to the left while the lowest-lying hole states are shifted to the right. Note that the hole states are visibly more polarized due to their greater mass.

\section{B. Density-matrix formalism}

To describe the dynamics of carriers in a quantum well, we define a density matrix in terms of the quantumwell eigenstates described in Sec. III A. $a_{n, \mathbf{k}}^{\alpha^{\dagger}}(t)$ and $a_{n, \mathbf{k}}^{\alpha}(t)$ are the Heisenberg operators that create and de- stroy electrons in a state $\varphi_{n, \mathbf{k}}^{\alpha}(\mathbf{r})$, and $\mathbf{k}$ represents the vector in the plane of the quantum well. The density matrix $(\mathrm{DM})$ is defined as

$$
N_{n m, \mathbf{k}}^{\alpha \beta}(t) \equiv\left\langle a_{n \mathbf{k}}^{\alpha^{\dagger}}(t) a_{m \mathbf{k}}^{\beta}(t)\right\rangle
$$

where \langle\rangle denotes the statistical average over the current nonequilibrium state of the system. Writing out the density matrix in terms of its $\alpha$ and $\beta$ components, and introducing some notation for the components of the density matrix, we have

$$
\begin{aligned}
N_{n m, \mathbf{k}}^{\alpha \beta} & =\left[\begin{array}{lll}
\left\langle a_{n \mathbf{k}}^{c \dagger}(t) a_{m \mathbf{k}}^{c}(t)\right\rangle & \left\langle a_{n \mathbf{k}}^{c \dagger}(t) a_{m \mathbf{k}}^{h}(t)\right\rangle & \left\langle a_{n \mathbf{k}}^{c^{\dagger}}(t) a_{m \mathbf{k}}^{l}(t)\right\rangle \\
\left\langle a_{n \mathbf{k}}^{h^{\dagger}}(t) a_{m \mathbf{k}}^{c}(t)\right\rangle & \left\langle a_{n \mathbf{k}}^{h^{\dagger}}(t) a_{m \mathbf{k}}^{h}(t)\right\rangle & \left\langle a_{n \mathbf{k}}^{h^{\dagger}}(t) a_{m \mathbf{k}}^{l}(t)\right\rangle \\
\left\langle a_{n \mathbf{k}}^{l^{\dagger}}(t) a_{m \mathbf{k}}^{c}(t)\right\rangle & \left\langle a_{n \mathbf{k}}^{l^{\dagger}}(t) a_{m \mathbf{k}}^{h}(t)\right\rangle & \left\langle a_{n \mathbf{k}}^{l^{\dagger}}(t) a_{m \mathbf{k}}^{l}(t)\right\rangle
\end{array}\right] \\
& \equiv\left[\begin{array}{lll}
n_{n m, \mathbf{k}}^{c}(t) & p_{n m, \mathbf{k}}^{c h}(t) & p_{n m, \mathbf{k}}^{c l}(t) \\
p_{n m, \mathbf{k}}^{c h}(t)^{*} & n_{n m, \mathbf{k}}^{h}(t) & p_{n m, \mathbf{k}}^{h l}(t) \\
p_{n m, \mathbf{k}}^{c l}(t)^{*} & p_{n m, \mathbf{k}}^{h l}(t)^{*} & n_{n m, \mathbf{k}}^{l}(t)
\end{array}\right]
\end{aligned}
$$

The interband components of the density matrix, $p_{n m, \mathbf{k}}^{\alpha \beta}(t)$, describe the coherence between different species of carriers, $\alpha$ and $\beta$, in subbands $n$ and $m$, respectively. They are related to the optical polarization. The intraband components of the density matrix, $n_{n m, \mathbf{k}}^{\alpha}(t)$, describe correlations between different subbands of the same carrier type if $n \neq m$. The diagonal components of the density matrix, $n_{n n, \mathbf{k}}^{\alpha}(t)$ represent the number of electrons in the quantum-well state $\varphi_{n, \mathbf{k}}^{\alpha}(\mathbf{r})$.

\section{Bloch equations}

The density matrix obeys the general equation of motion $^{2,10}$

$$
-i \hbar \frac{\partial N_{n m, \mathbf{k}}^{\alpha \beta}(t)}{\partial t}=\left\langle\left[H_{\mathbf{k}}, N_{n m, \mathbf{k}}^{\alpha \beta}(t)\right]\right\rangle,
$$

where the square brackets denote the commutator. The Hamiltonian operator for a quantum well interacting with a plane-polarized radiation field incident along $z$ and polarized along $x$ is

$$
\begin{aligned}
H_{\mathbf{k}}= & \sum_{\alpha n} E_{n}^{\alpha}(k) a_{n \mathbf{k}}^{\alpha^{\dagger}}(t) a_{n \mathbf{k}}^{\beta}(t) \\
& -E^{\text {opt }}(t) \sum_{\alpha \beta, n m} d_{n m, \mathbf{k}}^{\alpha \beta} a_{n \mathbf{k}}^{\alpha^{\dagger}}(t) a_{m \mathbf{k}}^{\beta}(t),
\end{aligned}
$$

where $E^{\text {opt }}(t)$ is the time-dependent optical electric field (with unit polarization vector $\mathbf{x}$ ), and $d_{n m, \mathbf{k}}^{\alpha \beta}$, the projection of the electron dipole moment along $\mathbf{x}$, is

$$
d_{n m, \mathbf{k}}^{\alpha \beta}=\int d \mathbf{r} \varphi_{n \mathbf{k}}^{\alpha}(\mathbf{r}) \operatorname{ex} \varphi_{m \mathbf{k}}^{\beta}(\mathbf{r}) .
$$

Since we are interested in pumping the quantum well 
with optical pulses with interband photon energy, we neglect intraband optical transitions (i.e., dipole transitions for which $\alpha=\beta$ ). In the effective-mass approximation, the interband dipole matrix elements are given by

$$
d_{n m}^{\alpha \beta}=\int d z F_{n}^{\alpha}(z)^{*} F_{m}^{\beta}(z) \int d \mathbf{r} u^{\alpha}(\mathbf{r})^{*} e x u^{\beta}(\mathbf{r}) .
$$

The dipole matrix elements for optical transitions are seen to be proportional to the product of the overlap integral between the envelope functions $F_{n}^{\alpha}(z)$ and $F_{m}^{\beta}(z)$ and the dipole matrix element between the bulk Bloch states $u^{\alpha}(\mathbf{r})$ and $u^{\beta}(\mathbf{r})$. The conduction-band bulk Bloch state $(\alpha=c)$ is $s$ like, and the valence-band bulk Bloch states $(\alpha=h, l)$ are $p$ like. Consequently, within our approximations for the valence bands the dipole transitions between heavy- and light-hole states are forbidden by symmetry. A more realistic calculation would include coupling of the valence bands to the lowest as well as higher conduction bands, which would make these matrix elements small but nonzero away from the zone center. $^{18,19}$ We neglect this effect, but point out that it can be important in other experiments such as infrared absorption in $p$-doped materials. ${ }^{20}$ The $p$-like bulk Bloch states with $x, y$, and $z$ symmetry (sixfold degenerate if we include spin) are split by spin-orbit interaction into a pair of $J=\frac{3}{2}, m= \pm \frac{3}{2}$ heavy-hole states and a pair of $J=\frac{3}{2}$, $m= \pm \frac{1}{2}$ light-hole states. ${ }^{21}$ (The $J=\frac{1}{2}, m= \pm \frac{1}{2}$ spin-split states are far away in energy and we ignore them.) The nonvanishing dipole matrix elements are ${ }^{21}$

$$
\int d \mathbf{r} u^{c}(\mathbf{r})^{*} e x u^{h}(\mathbf{r})=\frac{e}{\sqrt{2}} d_{0}
$$

and

$$
\int d \mathbf{r} u^{c}(\mathbf{r})^{*} \operatorname{exu}^{l}(\mathbf{r})=\frac{e}{\sqrt{6}} d_{0}
$$

where $d_{0}=\langle s|x| x\rangle=0.7 \mathrm{~nm}$ is the reduced dipole matrix element (along $x$ ) between the bulk conduction Bloch state with $s$-like symmetry and the valence Bloch state with $x$-like symmetry. For initial and final states from the same band, the matrix element (10) is simply determined by the envelope functions. Physically, such interband matrix elements describe movement of particles in their respective bands which will play an important role in further analysis.

Note that in the absence of the symmetry-breaking dc field, the overlap integrals in (11) would be zero for all transitions except $m=n$ [strictly speaking, this is only so in an infinite well at $\mathbf{k}=\mathbf{0}$, otherwise the transitions between the states of the same parity (e.g., $m=n+2$ ) become weakly allowed; however, such transitions do not affect the intraband dipole moment, so they are not essential to our analysis]. The dc field breaks the $\Delta n=0$ selection rule, so that each state in the valence band becomes coupled to more than one state in the conduction band, and vice versa. The presence of such crisscrossing transitions considerably complicates the description of optical excitation in such systems. These off-diagonal transitions are usually left out because in perturbation theory the overlap integrals with $\Delta n \neq 0$ are first order in the dc field, i.e., smaller than the diagonal $\Delta n=0$ elements which are of zero order. However, as we shall see below, these transitions are absolutely crucial to the dynamics of charge transport, and are necessary to describe the crossover from 2D to 3D behavior.

If we substitute the Hamiltonian into the equation of motion, we obtain the Bloch equations of motion for the components of the density matrix

$$
\begin{aligned}
\frac{\partial N_{n m, \mathbf{k}}^{\alpha \beta}}{\partial t}= & i\left[\frac{E_{n}^{\alpha}(k)-E_{m}^{\beta}(k)}{\hbar}\right] N_{n m, \mathbf{k}}^{\alpha \beta} \\
& +i \frac{E^{\mathrm{opt}}(t)}{\hbar} \sum_{\gamma j}\left(N_{n j, \mathbf{k}}^{\alpha \gamma} d_{j m}^{\gamma \beta}-d_{n j}^{\alpha \gamma} N_{j m, \mathbf{k}}^{\gamma \beta}\right) .
\end{aligned}
$$

In the simple model we are considering, the equations for different $k$ are completely decoupled and can be solved independently. The Coulomb interaction or the electron-phonon interaction would couple different $k$, making the problem extremely difficult to solve. There is a considerable body of work on incorporating the Coulomb interaction in the Bloch equations for both bulk and quantum-confined systems. ${ }^{13,22}$ However, for quantum wells in the electric field ${ }^{13}$ this was done in the diagonal approximation, i.e., neglecting the off-diagonal elements of the density matrix. In this paper, we neglect the Coulomb interactions, concentrating instead on a full quantum-mechanical description of the noninteracting electron and hole dynamics. In this sense, our approach is very similar to that of Ref. 2 .

From the Bloch equations, we can extract equations of motion for the various components of the density matrix. For the time-dependent optical field, we introduce a real electric field

$$
E^{\mathrm{opt}}(t)=E_{0} f(t) \operatorname{Re}\left(e^{-i \omega t}\right)
$$

The frequency $\omega$ is the central frequency of the pulse describing the rapid oscillations of the electric field, and $f(t)$ is a slowly varying envelope function that describes the pulse shape. For the pulse shape function, we assume a Gaussian pulse shape of the form

$$
f(t)=\exp \left[-\left(t / \tau_{p}\right)^{2}\right]
$$

where $\tau_{p}$ is the temporal width of the pulse. [Note that the full width at half maximum (FWHM) of such a pulse is $1.665 \tau_{p}$.]

We adopt the rotating wave approximation (RWA), and keep only those terms in the Bloch equations which are close to resonance with the free oscillations of the density matrix. We also factor out the rapid oscillations in $p_{n m, \mathbf{k}}^{c h}(t)$ and $p_{n m, \mathbf{k}}^{c l}(t)$ by introducing two variables

$$
\begin{aligned}
& p_{n m, \mathbf{k}}^{c h}(t) \equiv \widetilde{p}_{n m, \mathbf{k}}^{c h}(t) e^{i \omega t} \\
& p_{n m, \mathbf{k}}^{c l}(t) \equiv \widetilde{p}_{n m, \mathbf{k}}^{c l}(t) e^{i \omega t}
\end{aligned}
$$

Substituting these expressions into the Bloch equations and factoring out the term $e^{i \omega t}$, we obtain the following six equations for the components of the density matrix: 


$$
\begin{aligned}
& \frac{\partial n_{n m, \mathbf{k}}^{c}(t)}{\partial t}=i\left[\frac{E_{n}^{c}(k)-E_{m}^{c}(k)}{\hbar}\right) n_{n m, \mathbf{k}}^{c}(t)-\left\{\frac{\left(1-\delta_{n m}\right) n_{n m, \mathbf{k}}^{c}(t)}{\tau_{c}}\right\} \\
& +i \frac{E_{0} f(t)}{\hbar} \sum_{j}\left[\widetilde{p}_{n j, \mathbf{k}}^{c h}(t) d_{m j}^{c h}-d_{n j}^{c h} \widetilde{p}_{m j, \mathbf{k}}^{c h}(t)^{*}\right]+i \frac{E_{0} f(t)}{\hbar} \sum_{j}\left[\widetilde{p}_{n j, \mathbf{k}}^{c l}(t) d_{m j}^{c l}-d_{n j}^{c l} \widetilde{p}_{m j, \mathbf{k}}^{c l}(t)^{*}\right], \\
& \frac{\partial n_{n m, \mathbf{k}}^{h}(t)}{\partial t}=\left(\frac{E_{n}^{h}(k)-E_{m}^{h}(k)}{\hbar}\right) n_{n m, \mathbf{k}}^{h}(t)-\left\{\frac{\left(1-\delta_{n m}\right) n_{n m, \mathbf{k}}^{h}}{\tau_{h}}\right\}+i \frac{E_{0} f(t)}{\hbar} \sum_{j}\left[\widetilde{p}_{j n, \mathbf{k}}^{c h}(t)^{*} d_{j m}^{c h}-d_{j n}^{c h} \widetilde{p}_{j m, \mathbf{k}}^{c h}(t)\right], \\
& \frac{\partial n_{n m, \mathbf{k}}^{l}(t)}{\partial t}=i\left(\frac{E_{n}^{l}(k)-E_{m}^{l}(k)}{\hbar}\right) n_{n m, \mathbf{k}}^{l}(t)-\left\{\frac{\left(1-\delta_{n m}\right) n_{n m, \mathbf{k}}^{l}(t)}{\tau_{l}}\right\}+i \frac{E_{0} f(t)}{\hbar} \sum_{j}\left[\widetilde{p}_{j n, \mathbf{k}}^{c l}(t)^{*} d_{j m}^{c l}-d_{j n}^{c l} \widetilde{p}_{j m}^{c l}(t)\right], \\
& \frac{\partial \widetilde{p}_{n m, \mathbf{k}}^{c h}(t)}{\partial t}=i\left(\frac{E_{n}^{c}(k)-E_{m}^{h}(k)-\hbar \omega}{\hbar}\right) \widetilde{p}_{n m, \mathbf{k}}^{c h}(t)-\left\{\frac{\widetilde{p}_{n m, \mathbf{k}}^{c h}(t)}{\tau_{d}}\right\} \frac{E_{0} f(t)}{\hbar} \sum_{j}\left[n_{n j, \mathbf{k}}^{c}(t) d_{j m}^{c h}-d_{n j}^{c h} n_{j m, \mathbf{k}}^{h}(t)-d_{n j}^{c l} p_{m j}^{h l}(t)^{*}\right], \\
& \frac{\partial \widetilde{p}_{n m, \mathbf{k}}^{c l}(t)}{\partial t}=i\left(\frac{E_{n}^{c}(k)-E_{m}^{l}(k)-\hbar \omega}{\hbar}\right) \widetilde{p}_{n m, \mathbf{k}}^{c l}(t)-\left\{\frac{\widetilde{p}_{n m, \mathbf{k}}^{c l}(t)}{\tau_{d}}\right\} i \frac{E_{0} f(t)}{\hbar} \sum_{j}\left[n_{n j, \mathbf{k}}^{c}(t) d_{j m}^{c l}-d_{n j}^{c l} n_{j m, \mathbf{k}}^{l}(t)-d_{n j}^{c h} p_{j m, \mathbf{k}}^{h l}(t)\right], \\
& \frac{\partial p_{n m, \mathbf{k}}^{h l}(t)}{\partial t}=i\left(\frac{E_{n}^{h}(k)-E_{m}^{l}(k)}{\hbar}\right) p_{n m, \mathbf{k}}^{h l}(t)-\left\{\frac{p_{n m, \mathbf{k}}^{h l}(t)}{\tau_{d}}\right\}+i \frac{E_{0} f(t)}{\hbar} \sum_{j}\left[\widetilde{p}_{j n, \mathbf{k}}^{c h}(t)^{*} d_{j m}^{c l}-d_{j n}^{c h} \widetilde{p}_{j m, \mathbf{k}}^{c l}(t)\right] .
\end{aligned}
$$

The terms in curly brackets are dephasing terms which have been added phenomenologically to allow for the effects of electron-hole-phonon and carrier-carrier ( $e-h$, $e-h, h-h)$ collisions. We allow for different relaxation times for interband and intraband polarizations, but do not include relaxation terms for the densities (longitudinal relaxation) since their relaxation is typically much slower than that of the polarizations. In all numerical calculations below, we have assumed $100 \mathrm{fs}$ as the interband dephasing time, and 1 ps for intraband dephasing. ${ }^{3,4}$

This is a set of coupled ordinary differential equations that can be solved using an adaptive stepsize RungeKutta routine. For boundary conditions, we assume that in the remote past the conduction subbands are empty and the valence subbands are occupied, so that $n_{n m, \mathbf{k}}^{c}(t \rightarrow-\infty)=0, \quad n_{n m, \mathbf{k}}^{h}(t \rightarrow-\infty)=\delta_{n, m}, \quad$ and $n_{n m, \mathbf{k}}(t \rightarrow-\infty)=\delta_{n, m}$. The polarizations are assumed to vanish so that $\widetilde{p}_{n m, \mathbf{k}}^{c h}(t \rightarrow-\infty)=0, \widetilde{p}_{n m, \mathbf{k}}^{c l}(t \rightarrow-\infty)=0$, and $p_{n m, \mathbf{k}}^{h l}(t \rightarrow-\infty)=0$.

Note that in zero dc field this set of equations is greatly simplified since, in our model which neglects the band mixing, the density-matrix components with $n \neq m$ vanish because of the $\Delta n=0$ selection rule in our calculation. In a finite dc field, the off-diagonal terms of the DM are first order in the dc field and have to be kept in order to correctly describe the charge transport. Since we keep several tens of subbands (mostly in the heavy-hole band), we typically have to solve a few thousand equations for each $\mathbf{k}_{1}$. The number of equations to be solved is determined dynamically by the program. It depends primarily on well width and pulse duration. The more quantumwell states that lie within the excitation spectral width, the more equations that need to be solved.

\section{Observables}

Once the density matrix is known, we can calculate observable quantities such as charge density, dipole moment, and current densities.

\section{Charge density}

The simplest observable is the particle density which, at a fixed value of $k$, is defined in terms of field operators as

$$
P_{\mathbf{k}}(\mathbf{r}, t)=\left\langle\psi_{\mathbf{k}}^{\dagger}(\mathbf{r}, t) \psi_{\mathbf{k}}(\mathbf{r}, t)\right\rangle .
$$

In the basis of our effective-mass wave functions, the field operator is given by

$$
\psi_{\mathbf{k}}^{\dagger}(\mathbf{r}, t)=\sum_{\alpha n} \frac{e^{-i \mathbf{k} \cdot \mathbf{r}}}{\sqrt{A}} F_{n}^{\alpha}(z) u^{\alpha}(\mathbf{r}) a_{n}^{\alpha^{\dagger}}(t) .
$$

Inserting the field operator into the expression for the particle density, we obtain the relation between the particle density and the density matrix:

$$
\begin{aligned}
P_{\mathbf{k}}(\mathbf{r}, t)= & \sum_{\alpha \beta, n m} \frac{F_{n}^{\alpha}(z) F_{m}^{\beta}(z)}{A} \\
& \times u^{\alpha}(\mathbf{r}) u^{\beta}(\mathbf{r})\left\langle a_{n \mathbf{k}}^{\alpha \dagger}(t) a_{m \mathbf{k}}^{\beta}(t)\right\rangle \\
= & \sum_{\alpha \beta, n m} \frac{F_{n}^{\alpha}(z) F_{m}^{\beta}(z)}{A} u^{\alpha}(\mathbf{r}) u^{\beta}(\mathbf{r}) N_{n m, \mathbf{k}}^{\alpha \beta}(t) .
\end{aligned}
$$

Leaving out interband terms which rapidly oscillate in time and space, and averaging over the periodic part of the Bloch functions, we obtain 


$$
P_{\mathbf{k}}(\mathbf{r}, t)=\sum_{\alpha, n m} \frac{F_{n}^{\alpha}(z) F_{m}^{\alpha}(z)}{A} n_{n m, \mathbf{k}}^{\alpha}(t) .
$$

We can also write this result in terms of the charge density in a specific band. Thus

$$
P_{\mathbf{k}}(\mathbf{r}, t)=\sum_{\alpha, n} \rho_{n, \mathbf{k}}^{\alpha}(\mathbf{r}, t),
$$

where the charge density of species $\alpha$ in subband $n$ with wave vector $k$ is defined as

$$
\rho_{n, \mathbf{k}}^{\alpha}(\mathbf{r}, t) \equiv \sum_{m} \frac{F_{n}^{\alpha}(z) F_{m}^{\alpha}(z)}{A} \operatorname{Re}\left(n_{n m, \mathbf{k}}^{\alpha}(t)\right) .
$$

The total charge density is obtained by summing over $k$ in the plane of the well. Since different $k$ 's are uncorrelated in our formalism, the result is simply

$$
\begin{aligned}
\rho_{n}^{\alpha}(z, t) & =\sum_{\mathbf{k}} \rho_{n, \mathbf{k}}^{\alpha}(z, t) \\
& =\frac{A}{2 \pi} \int_{0}^{\infty} d k k \rho_{n, k}^{\alpha}(z, t) \\
& =\sum_{m} F_{n}^{\alpha}(z) F_{m}^{\alpha}(z) \frac{1}{2 \pi} \times \int_{0}^{\infty} d k k \operatorname{Re}\left(n_{n m, k}^{\alpha}(t)\right) .
\end{aligned}
$$

\section{Dipole moment}

At a fixed value of $k$, the electron dipole moment in the direction $z$ of the dc field is defined in terms of charge density as

$$
\mathbf{d}_{\mathbf{k}}(t)=\int d \mathbf{r} P_{\mathbf{k}}(\mathbf{r}, t) e \mathbf{r}=\sum_{\alpha, n m} n_{n m, \mathbf{k}}^{\alpha}(t) D_{n m}^{\alpha} \hat{z},
$$

where

$$
D_{n m}^{\alpha}=\int d z F_{n}^{\alpha}(z) e z F_{m}^{\alpha}(z)
$$

is just the dipole matrix element between the slowly varying envelope functions. Consistent with the approximations made in Eq. (27), we have neglected the rapidly varying interband piece. Finally, the total dipole moment per unit area is obtained by summing over $k$ and dividing by the area of the quantum well:

$$
\mathbf{d}(t)=\frac{1}{A} \sum_{\mathbf{k}} \mathrm{d}_{\mathbf{k}}=\frac{1}{2 \pi} \int_{0}^{\infty} d k k \sum_{\alpha n m} n_{n m, k}^{\alpha}(t) D_{n m}^{\alpha} \hat{z} .
$$

Note that the off-diagonal intraband matrix elements are zero order in the dc field, while the diagonal ones are first order. The first-order contributions to the dipole moment are then formed in two ways: diagonal dipole matrix element (first order) times a diagonal DM component (zero order), or off-diagonal matrix element (zero order) times an off-diagonal DM element (first order). In the usual polarized-pair calculations, only the diagonal contribution is taken into account. We will examine the role of the off-diagonal terms below.

\section{Current density}

The current density is just the time derivative of the displacement (31). Thus

$$
\mathrm{j}(t)=\frac{d}{d t}\left\{\frac{\mathrm{d}(t)}{A w}\right\} .
$$

This current is time dependent and produces transient electromagnetic radiation which is experimentally accessible. . $^{3,9-12}$ The electric field of the radiated wave is proportional to the time derivative of (34).

\section{RESULTS}

Using the above formalism, we are able to calculate the coherent response of quantum wells for different well geometries and excitation conditions. Because of the large number of parameters involved (well width $w$, pulse duration $\tau_{p}$, photon energy $\hbar \omega$, dc field $\mathbf{E}$, and relaxation times) we cannot explore all of the parameter space, but only present results that are representative of the several physically different regimes. The first case we consider is that of narrow wells and/or long pulses. Here the energy width of the excitation spectrum is smaller than the spacing of quantized levels (we concentrate on the conduction band for simplicity). In this case, electrons from a single valence-band state are optically coupled to no more than one final state in the conduction band [Fig. 3(a)]. Consequently, there is no coherence between different conduction-band states, and the charge density is an incoherent sum of densities of each populated state. As is seen in Fig. 3(b), the charge-density distribution in this case simply scales with the total number of carriers preserving its shape which is determined by a weighted sum of wave functions squared for each populated level. The time dependence of the dipole moment in this case [Fig. 3(c)] follows the number of carriers, since there is a constant dipole moment per photogenerated electronhole pair.

In this confined limit the quantum well behaves as a collection of independent two-level systems whose observables are added incoherently according to the original polarized-pairs argument. ${ }^{9,13}$ The experimental conditions of Refs. 3 and 4 correspond to this regime. Below we will examine the quantitative conditions for this and other regimes in more detail.

For shorter pulses and/or wider wells the energy width of the excitation spectrum becomes comparable to the level spacing, so that multiple transitions from valenceband states become energetically possible [Fig. 4(a)]. If two or more conduction-band states become optically coupled to the same state in the valence band, the conduction-band wave function is a coherent superposition of their wave functions. In density-matrix language, this means that there are nonzero off-diagonal elements $n_{n m}^{c}$ of the density matrix. The corresponding terms in the expression for the charge density (30) and other observables (31) and (34) oscillate at the difference frequencies. These oscillations (quantum beats) are clearly seen in the time-dependent charge density [Fig. 4(b)] and the dipole moment [Fig. 4(c)]. In this case, the carriers no longer occupy definite eigenstates, instead they form wave packets that can move inside the well. This regime is easily achieved in asymmetric double-well structures, ${ }^{2,4}$ where the level spacing can be made very small. 

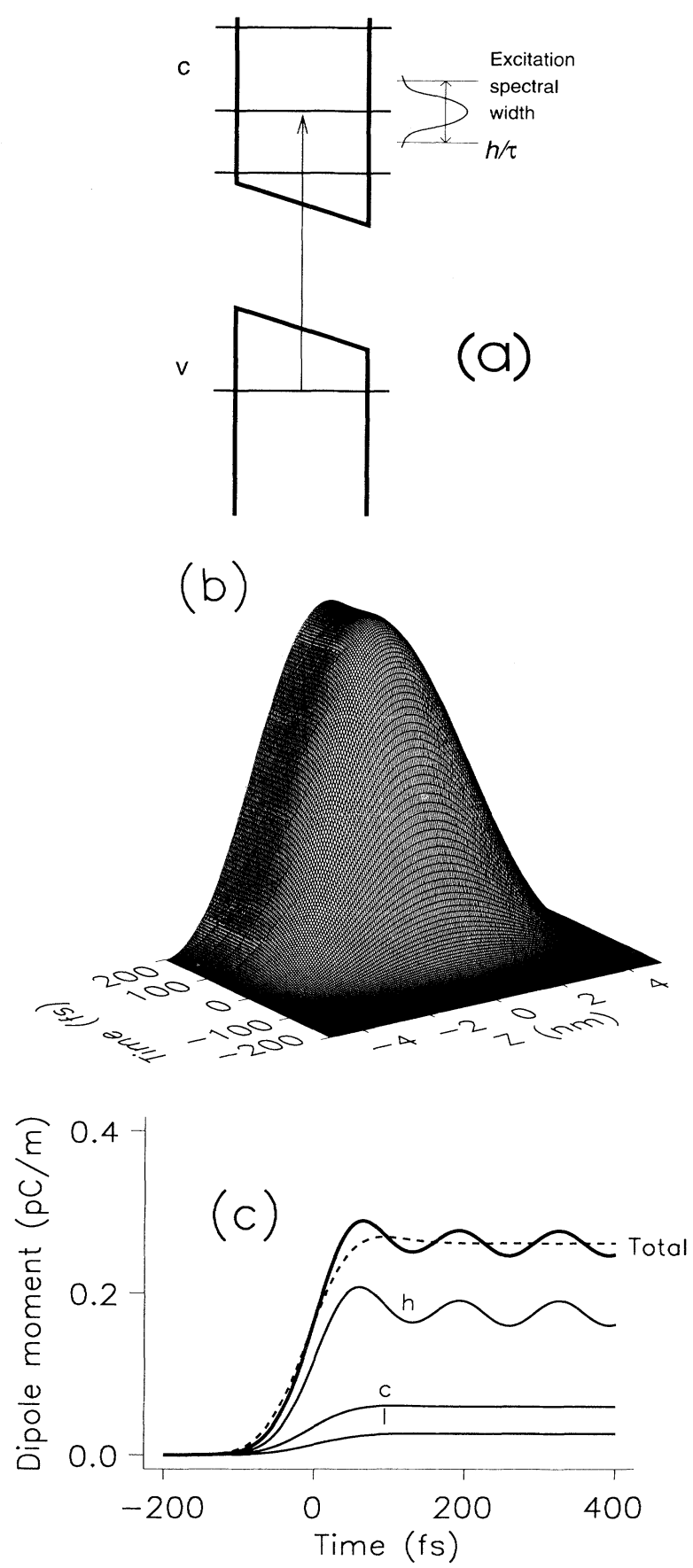

FIG. 3. (a) A schematic representation of optical transitions in a narrow (10 $\mathrm{nm}$ ) well. The energy splitting between the levels is greater than the spectral width of the excitation. There is only one final state for transitions from any given valence-band state. (b) Time-dependent charge density for conduction electrons in a $10-\mathrm{nm}$ quantum well in a $50-\mathrm{kV} / \mathrm{cm}$ field excited by a $100-\mathrm{fs}$ pulse. The central frequency of the excitation is $1.55 \mathrm{eV}$ (about $100 \mathrm{meV}$ above the band gap). The shape of the distribution does not change in time because it is an incoherent sum of wave function squared for the levels involved in the transition. (c) Time-dependent polarization. The dashed line is the diagonal component of the polarization [neglecting off-diagonal terms in (33)]. The polarization follows the diagonal component closely. Note a small quantum beat contribution due to heavy holes.
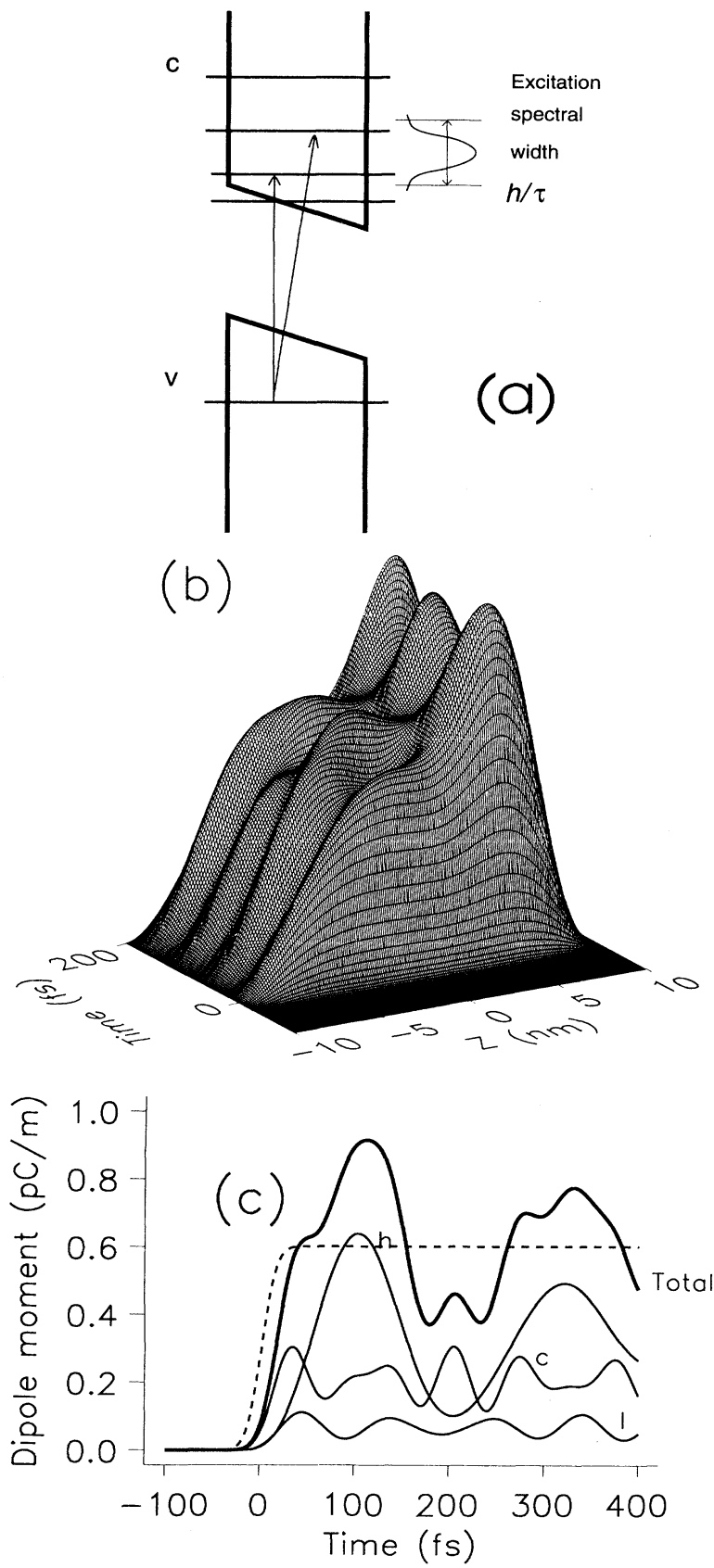

FIG. 4. (a) A schematic representation of optical transitions in a wider $(20 \mathrm{~nm})$ well. The energy splitting between the levels is now comparable to the spectral width of the excitation, so that multiple transitions from a given valence-band state become possible. (b) Time-dependent charge density for conduction electrons in a $20-\mathrm{nm}$ quantum well in a $50-\mathrm{kV} / \mathrm{cm}$ field excited by a 25 -fs pulse (central frequency $1.50 \mathrm{eV}$ ). Initially, the charge density is practically uniform across the well. At later times, the density exhibits oscillatory motion (quantum beats) due to interference between different levels involved in the superposition. (c) Time-dependent polarization contains quantum beats created by all three carrier species. The beats for conduction electrons (c) contain multiple frequencies. Note the delay between the diagonal component which follows the total density (dashed line) and the full result (solid line), which is due to initial flatness of the density distribution. 
We shall call this regime the quantum beat limit. Let us note that the magnitude of the quantum beat component in the dipole moment is generally comparable to the diagonal polarized-pair component: both are first order in the dc field [see the discussion after Eq. (33)]. Therefore, for shorter pulses whose spectrum covers more than one level, the polarized-pairs concept is not applicable even for low dc fields (even for zero dc fields there will be some coupling owing to the previously discussed violations of the $\Delta n=0$ selection rule).

For even shorter pulses, the number of levels involved in the quantum beats becomes large [Fig. 5(a)], and the beating pattern in the charge density becomes more complicated with many different frequencies present [Fig. 5(b)]. The time evolution of the charge density in this limit is quite different from the confined case of Fig. 3(b) - the charge density is initially uniform inside the well. This is because initially there is a very large number of levels contributing to the charge density (30), and the oscillatory components of their wave functions squared tend to cancel out. Because of this effect, there is a noticeable delay between the rise of the density [represented by a dashed line in Fig. 5(c)] and the rise of the dipole moment - in this regime, the dipole moment does not follow the number of carriers instantaneously. Let us also point out that in spite of the large number of levels involved in the formation of the wave packets shown in Fig. 5(b), the dynamics of the dipole moment turns out to be quite simple with almost monochromatic beats for each carrier species. We will call this last regime the classical limit for reasons explained below.

These different types of behavior can be very naturally understood in terms of the transient localization concept. As was demonstrated in Ref. 10, in bulk systems one can describe the optical generation of carriers in a dc electric field as a process of creation of electronic wave packets which then propagate from the point of creation. The size of these wave packets, $l_{c}$, is independent of the dc field and is given by

$$
l_{c}=\tau_{p} \cdot \mathbf{v}_{0}
$$

where $\tau_{p}$ is the pulse duration, and $\mathbf{v}_{0}$ is a characteristic velocity the carriers are created with (it is given by the condition $\mathbf{v}_{0}=\hbar \mathbf{k}_{0} / m_{\alpha}$, where $\mathbf{k}_{0}$ is determined by energy and momentum conservation requirements: $\hbar \omega-E_{g}=\hbar \mathbf{k}_{0}^{2} / 2 \mu$ ). For, e.g., a 100 -fs pulse and $\Delta=\hbar \omega-E_{g}=100 \mathrm{meV}$ the size of conduction-electron wave packets is about $50 \mathrm{~nm}$. The size of the wave packets is a measure of the spatial extent of electronic wave functions. We will call this quantity the coherence length. This should be distinguished from the phase-breaking length, which is the distance the wave packets can travel before the relaxation destroys them $\left(l_{\mathrm{ph}}=\tau_{\mathrm{rel}} \mathbf{v}_{0}\right)$.

The different transport regimes identified above correspond to different relations between the coherence length and the well width. For the perfectly confined case of Fig. $2, l_{c} \gg w$, the spatial extent of electronic wave functions in the bulk would have been much greater than the width of the well. The wave packet has to adjust the boundary conditions imposed by the well, which pro-
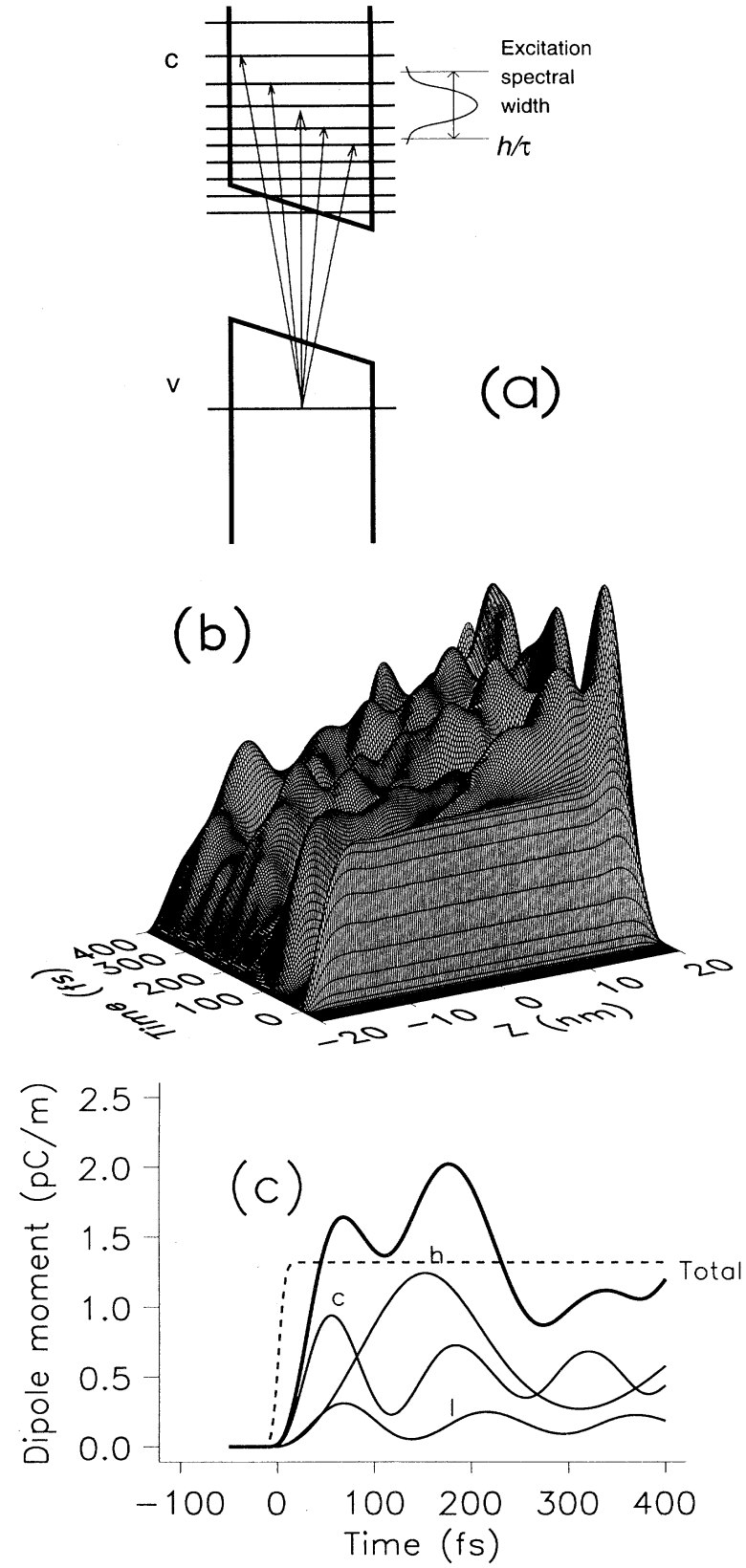

FIG. 5. (a) Transition diagram for a wide well with a large number of levels covered by excitation spectrum. (b) Timedependent charge density of conduction electrons in a wide 40$\mathrm{nm}$ well in a $50-\mathrm{kV} / \mathrm{cm}$ field. The excitation pulse is short (10 fs) and centered at $1.50 \mathrm{eV}$. The charge density is initially uniform across the well and exhibits very complex beating pattern at later times. (c) The polarization for all three carrier species is dominated by oscillations, and there is an appreciable delay between the rise of the density (dashed line) and that of the polarization.

duces well-defined energy levels.

The classical limit of Fig. 5 corresponds to the opposite relation between these two lengths: $l_{c}<w$. In this limit, the pulse creates wave packets that are much smaller than the well. Wave packets that are created more than $l_{c}$ away from either wall behave exactly as they would in 
the bulk until they approach the walls. Therefore, in this regime the photocarriers in the quantum well behave like an ensemble of classical particles that are created uniformly in the well with corresponding classical velocities, and then move in the applied field according to classical laws of motion. This is the reason for the initial flatness of the density distribution which is evident in Figs. 4(b) and 5(b). In a confined sample, the carriers cannot separate indefinitely because they are reflected by the walls. Therefore, even for classical particles, one can expect an oscillatory time dependence of the dipole moment instead of a monotonic $t^{2}$ growth characteristic of bulk samples [Fig. 1(c)]. For the ensemble of classical particles it can be shown that the average dipole moment should exhibit quasimonochromatic oscillations with the period equal to the maximum period of classical motion for all particles in the ensemble. This explains why there are no multiple frequencies present in the dipole moment dynamics shown in Fig. 5(c).

In Fig. 6 we plot the time-dependent dipole moment calculated for an ensemble of classical particles for the excitation conditions of Fig. 5. We introduce point particles randomly across the width of the well according to temporal profile of the excitation, and assign them a randomly directed velocity (35). There is an excellent overall agreement between the classical model of Fig. 6 and the full quantum-mechanical calculation displayed in Fig. 5. The agreement is especially good for the heavy holes, whose coherence length is about 50 times smaller than the well width in this example. For the electrons the coherence length is about $\frac{1}{10}$ the well width, but still the agreement between quantum-mechanical and classical calculations is very good at early times.

For a given value of detuning, we can represent different possible regimes on a phase diagram like the one shown in Fig. 7. This is a plot of the coherence length $l_{c}$ for conduction electrons and heavy holes as a function of pulse duration (we do not plot the line for light holes for simplicity). The region far below the solid line corresponds to pulse duration well width combinations for which a confined behavior like the one shown in Fig. 3 is

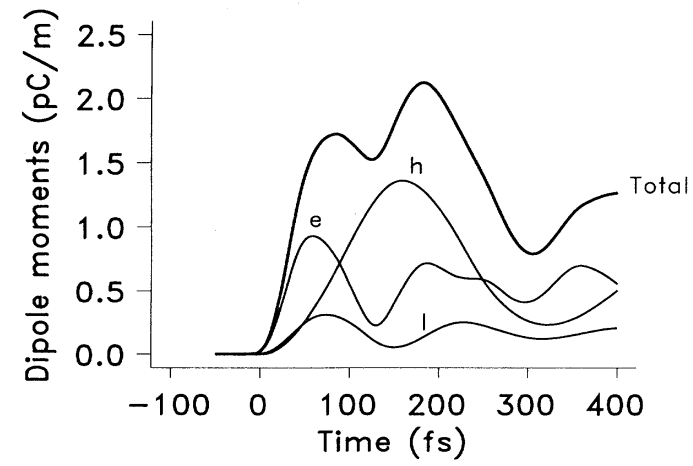

FIG. 6. The time-dependent polarization for an ensemble of classical particles in a biased well with the same parameters as in Fig. 5. The classical polarization is very close to that shown in Fig. 5(c), especially at earlier times.

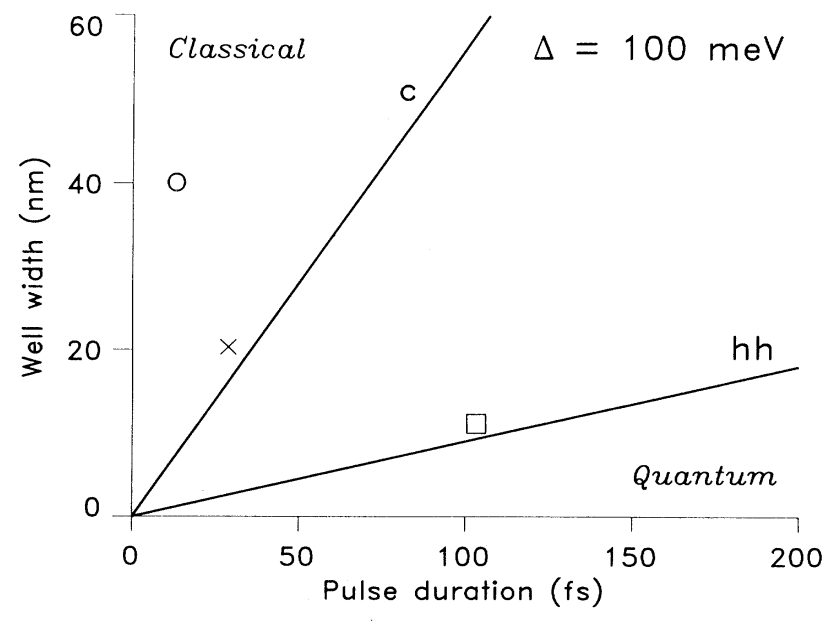

FIG. 7. Phase diagram for different excitation regimes. The straight lines represent the condition $l_{c}=w$ for conduction electrons and heavy holes. The upper left corner (wide wells and short pulses) corresponds to a classical regime (the conditions of Fig. 5 are represented by a circle), the lower right corner (narrow wells and long pulses) is the quantum-confined regime (the square represents Fig. 3). The intermediate quantum beat regime corresponds to the vicinity of the lines. The cross shows the conditions of Fig. 4, where the conduction electrons are in the quantum beats regime.

achieved. The region well above this line corresponds to the classical limit where the well behaves like a 3D system (Figs. 5 and 6). Finally, the region adjacent to the $w=l_{c}$ line represents the intermediate quantum beat regime shown in Fig. 4.

The relaxation can be included phenomenologically by limiting the coherence length by the phase-breaking length. For long pulses, the solid curves should approach the plateaus $w=l_{\text {ph }}$ which represent the well widths above which the wells can be considered 3D even in the steady state. This would correspond to the usual criterion of level spacing being equal to the broadening of the levels.

\section{CONCLUSIONS}

In Sec. III, we identified three distinct regimes for polarization dynamics in optically excited quantum wells. The first (quantum confined) regime corresponds to the conventional picture of polarized-pair creation,, 13 and occurs for long excitation pulses and narrow wells such that the condition $w \ll l_{c}$ is met (Fig. 3). In the second regime $w \sim l_{c}$, the carrier wave functions are formed by coherent superposition of a few eigenstates connected by the optical transition. This leads to quantum beats on difference frequencies. Such quantum beats have been previously predicted and observed ${ }^{2,4}$ in double-well structures where the level spacing can be made small. Finally, the third, classical regime is obtained when the number of levels involved in the superposition becomes large $\left(w>>l_{c}\right)$. We have shown that in this case the carriers 
behave as classical pointlike particles moving between the walls of the well in the applied dc field, so that the well behaves essentially as a $3 \mathrm{D}$ system.

The dc field applied to the well breaks the $\Delta n=0$ selection rule for optical transitions, so that in principle there is a nonzero optical matrix element between any state in the valence band and any state in the conduction band. For long pulses, most of these off-diagonal transitions are forbidden energetically. One can then retain only diagonal transitions, which considerably simplifies the problem and results in the standard polarized-pairs approach. ${ }^{4,9,13}$ However, this cannot be done for shorter pulses whose spectral width is large enough to cover several levels. When transitions from one initial state to many final states become possible, the carrier dynamics have to be described in terms of wave packets (coherent superpositions of the eigenstates) that can move inside the well [Figs. 4(b) and 5(b)] and produce quantum beats in the time-dependent polarization.

For still shorter pulses, the characteristic size of the wave packets [coherence length (35)] becomes smaller than the well, and the carriers start to behave like pointlike classical particles. This classical regime is very difficult to analyze in our formalism since the number of levels involved in the superpositions becomes quite large (several tens for the heavy holes). However, the carrier dynamics can be described in this case by modeling the photocarriers with an ensemble of classical particles (Fig. 6) which is orders of magnitude easier computationally.

As is clear from Fig. 7, for experiments done with 100fs pulses almost any quantum well is in the quantumconfined regime. This justifies the use of the diagonal approximation that was implicitly made in Refs. 4, 9, and 13 [although the heavy holes can exhibit quantum beats even for 100-fs pulses; see Fig. 3(c)]. At present, however, much shorter pulses up to $10 \mathrm{fs}$ are being used for quantum-well studies. ${ }^{23}$ For such short-pulse experiments, our predictions of quantum beats and possible classical transport motion of carriers become important. In particular, making the excitation pulses shorter does not necessarily make current transients shorter, since for short pulses the carriers can exhibit classical behavior and produce a transportlike current which no longer follows the pulse adiabatically. This is illustrated in Fig. 8, where we compare the calculated photocurrent with its adiabatic (diagonal) component. For the case shown in Fig. 8, the photocurrent does not follow the pulse adia-

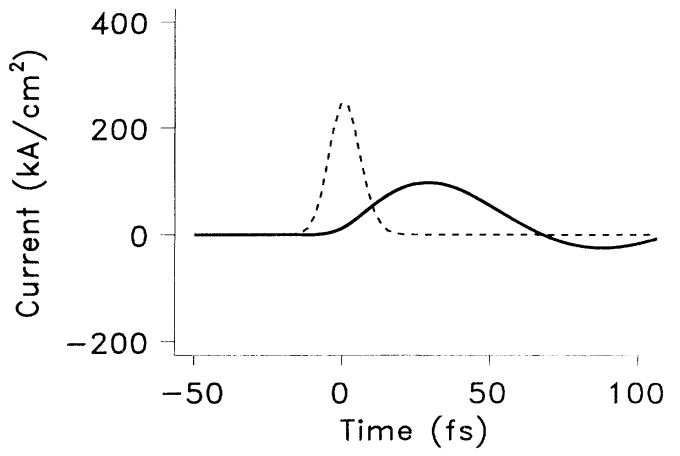

FIG. 8. A comparison of photocurrent calculated for a wide quantum well in the classical regime (solid line) with the result of the adiabatic following approximation that neglects the offdiagonal components of the density matrix. The parameters are those of Fig. 5. The duration of the photocurrent peak is much longer than the excitation pulse duration (10 fs).

batically, and changes on a much slower time scale. In fact the current oscillations in Fig. 8 (or the polarization oscillations in Fig. 5) are so slow that they can be detected by conventional dipole antennas. ${ }^{3,12}$

Our approach allows us to describe the dynamics of electronic wave packets in quantum wells. However, due to computational limitations we have to leave out a number of experimentally relevant effects. For near-band-gap excitation, excitonic effects should play a major role. Our formalism also neglects the above-barrier states (they are too numerous to include), while the results of Ref. 10 suggest that virtual population of these states can make a large adiabatic contribution to the polarization. In spite of these limitations, we believe that our formalism captures the essential features of the wave-packet dynamics in quantum wells.

\section{ACKNOWLEDGMENTS}

This work was supported by the U.S. Office of Naval Research through Grant No. N0091-JJ-1956, and by the National Science Foundation through Grant No. DMR8957382. C.J.S gratefully acknowledges support from the Alfred P. Sloan Foundation. We also wish to thank the Danish Research Academy and the Microelektronics Centret at DTU for support during part of this work.
${ }^{*}$ Permanent address: Department of Physics, Ohio State University, Columbus, OH 43210.

†Permanent address: Department of Physics, University of Florida, Gainesville, FL 32611.

${ }^{1}$ See a collection of review articles, in Hot Carriers in Semiconductor Nanostructures: Physics and Applications (Academic, New York, 1992).

${ }^{2}$ T. Kuhn, E. Binder, F. Rossi, A. Lohner, K. Rick, P. Leisching, A. Leitenstorfer, T. Elsaesser, and W. Stolz, in Coherent Optical Interactions in Semiconductors, edited by R. T. Phil- lips (Plenum, New York, 1994), p. 33.

${ }^{3}$ P. C. M. Planken, M. C. Nuss, W. H. Knox, D. A. B. Miller, and K. W. Goossen, Appl. Phys. Lett. 61, 2009 (1992); P. C. M. Planken, M. C. Nuss, I. Brener, K. W. Goossen, M. S. C. Luo, S. L. Chuang, and L. Pfeiffer, Phys. Rev. Lett. 69, 3800 (1992).

${ }^{4}$ M. C. Nuss, P. C. M. Planken, I. Brener, H. Roskos, M. S. Luo, and S. L. Chuang, Appl. Phys. B 58, 2216 (1994).

${ }^{5}$ T. Dekorsy, P. Leisching, K. Kohler, and H. Kurz, Phys. Rev. B 50, 8106 (1994). 
${ }^{6}$ J. Feldmann, K. Leo, J. Shah, D. A. B. Miller, J. E. Cunningham, S. Schmitt-Rink, T. Meier, G. von Plessen, A. Schultze, and P. Thomas, Phys. Rev. B 46, 7252 (1992).

${ }^{7}$ C. Waschke, H. G. Roskos, R. Schwedler, K. Leo, H. Kurz, and K. Kohler, Phys. Rev. Lett. 70, 3319 (1993).

${ }^{8} \mathrm{Ch}$. Kittel, Quantum Theory of Solids (Wiley, New York, 1963).

${ }^{9}$ S. L. Chuang, S. Schmitt-Rink, B. I. Greene, P. N. Saeta, and A. F. J. Levi, Phys. Rev. Lett. 68, 102 (1992).

${ }^{10}$ A. V. Kuznetsov and C. J. Stanton, Phys. Rev. B 48, 10828 (1993).

${ }^{11}$ X.-C. Zhang, B. B. Hu, J. T. Darrow, and D. H. Auston, Appl. Phys. Lett. 56, 1011 (1990); B. B. Hu, X.-C. Zhang, and D. H. Auston, Phys. Rev. Lett. 67, 2709 (1991).

${ }^{12}$ B. B. Hu, A. S. Weling, D. H. Auston, A. V. Kuznetsov, and C. J. Stanton, Phys. Rev. B 49, 2234 (1994).

${ }^{13}$ S. Schmitt-Rink, D. S. Chemla, and D. A. B. Miller, Adv. Phys. 38, 89 (1989).

${ }^{14}$ Y. Yafet and E. Yablonovich, Phys. Rev. B 43, 12480 (1991); E. Yablonovich, J. P. Heritage, D. E. Aspnes, and Y. Yafet, Phys. Rev. Lett. 63, 976 (1989).
${ }^{15}$ D. S. Chemla, D. A. B. Miller, and S. Schmitt-Rink, Phys. Rev. Lett. 59, 1018 (1987); M. Yamanishi, ibid. 59, 1014 (1987).

${ }^{16}$ K. W. Boer, Survey of Semiconductor Physics (Van Nostrand, New York, 1990), p. 219.

${ }^{17}$ J. M. Luttinger, Phys. Rev. 102, 1030 (1956); J. M. Luttinger and W. Kohn, ibid. 97, 869 (1955).

${ }^{18}$ Y. C. Chang and R. B. James, Phys. Rev. B 39, 672 (1989).

${ }^{19}$ M. S. C. Luo, S. L. Chuang, P. C. M. Planken, I. Brener, H. G. Roskos, and M. C. Nuss, IEEE J. Quantum Electron. QE30, 1478 (1994).

${ }^{20}$ B. F. Levine, S. D. Gunapala, J. M. Kuo, S. S. Pei, and S. Hui, Appl. Phys. Lett. 59, 1864 (1991).

${ }^{21}$ F. Bassani and G. Parravicini, Electronic States and Optical Transition in Solids (Pergamon, New York, 1975).

${ }^{22}$ M. Lindberg, R. Binder, and S. W. Koch, Phys. Rev. A 45, 1865 (1992); Y. Z. Hu, R. Binder, and S. W. Koch, Phys. Rev. B 47, 15679 (1993).

${ }^{23}$ B. B. Hu, E. A. de Souza, W. H. Knox, J. E. Cunningham, M. C. Nuss, A. V. Kuznetsov, and S. L. Chuang, Phys. Rev. Lett. 74, 1689 (1995). 